\section{Deep-UV Detector for HPLC with Light-Emitting Diode}

Stefan Schmid ${ }^{\mathrm{a}}$, Mirek Macka ${ }^{\mathrm{b}}$, and Peter C. Hauser ${ }^{\star a}$

${ }^{*}$ Correspondence: Prof. Dr. P. C. Hauser, Tel.: +4161267 1003, Fax: +4161267 1013, E-mail: peter.hauser@unibas.ch

aDepartment of Chemistry, University of Basel, Spitalstrasse 51, CH-4056 Basel bepartment of Chemical Sciences, Dublin City University, Glasnevin, Dublin 9, Ireland

\section{Keywords: Detector · HPLC · LED · UV}

Light-emitting diodes (LEDs) give off relatively narrow spectral bands whose wavelengths are directly related to the bandgap energy between the $\mathrm{p}$ - and $\mathrm{n}$-doped semiconducting materials employed. They were commercialised in the early 1970s and were first made available for the infrared and then the red region of the visible spectrum. Subsequently LEDs with ever shorter wavelengths, which are more difficult to produce due to the higher energies involved, have become available. At the same time it was also possible to increase the intensities to the point that they now become attractive as more efficient replacements for conventional light sources. Standard LEDs have emission bandwidths of about $30 \mathrm{~nm}$, matching well the absorption bandwidths of molecules. Thus the use of LEDs as radiation sources for spectrophotometric instrumentation, eliminating the need for monochromators, was suggested in 1973. LEDs now have found application in analytical instruments where compactness and low power consumption are required and low cost is desired, but where flexibility in wavelength setting is not needed.

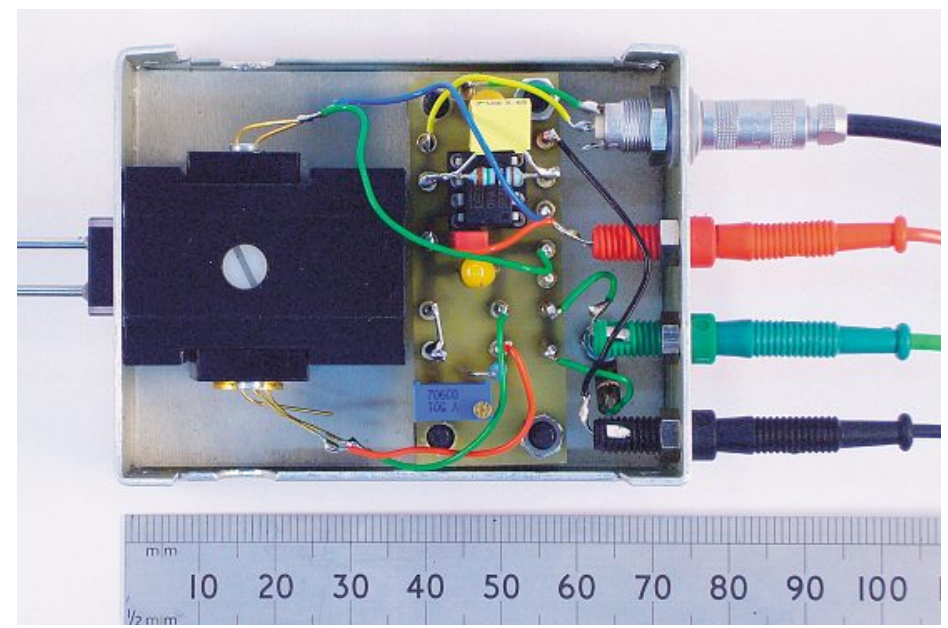

The flow-through cuvette is fixed in the black holder; the UV-LED and the photodiode are attached on its sides
Recently the wavelengths attained with LEDs have reached the deep UV-range below $300 \mathrm{~nm}$, and this opens up new possibilities. While the intensities are presently not adequate for excitation in fluorescence, absorbance measurements are feasible. This has allowed the construction of an unprecedented simple and compact detector for HPLC. For measuring the intensity of the UV-light, a special photodiode, which had been designed for UV-radiometry, was employed. The photocurrent was converted to an output voltage by using a simple operational amplifier in an integrated circuit package. The wavelength of $255 \mathrm{~nm}$ is useful for many aromatic compounds.

Test mixtures of benzoic acid, nitrobenzene, benzyl acetate and methyl benzoate were separated using an acetonitrile/water gradient elution program. Limits of detection of $750 \mathrm{ng} \cdot \mathrm{ml}^{-1}, 5.8 \mu \mathrm{g} \cdot \mathrm{ml}^{-1}$ and $12 \mu \mathrm{g} \cdot \mathrm{ml}^{-1}$ were obtained for nitrobenzene, benzoic acid, and methyl benzoate, respectively. These values correspond to the relative absorptivities of the compounds at $255 \mathrm{~nm}$ and are close to the detection limits obtained with conventional commercial detectors. The detection limit for benzyl acetate was not determined as the sensitivity for this compound is low, due to a poor spectral match. Further improvements in deep-UV LED technology in terms of intensity and shorter wavelengths can be expected. They will lead to lower limits of detection, which match or surpass those of conventional detectors, and to an even wider range of applications in absorbance measurements.

Received: September 5, 2008

\section{References}

H. Flaschka, C. McKeithan, R. Barnes, Anal. Lett. 1973, 6, 585.

S. Schmid, M. Macka, P. C. Hauser, Analyst 2008, 133, 465.

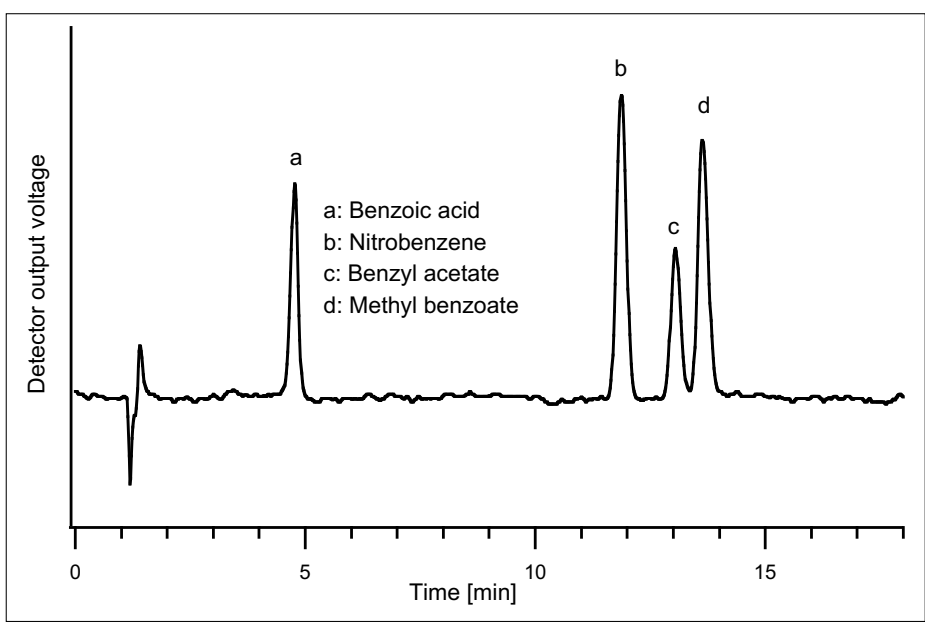

HPLC chromatogram of a test mixture 